\title{
ARTICLE OPEN Hydrogen blistering under extreme radiation conditions
}

\author{
Maciej Sznajder $\mathbb{D}^{1,2}$, Ulrich Geppert ${ }^{3}$ and Mirosław R. Dudek ${ }^{2}$
}

Metallic surfaces, exposed to a proton flux, start to degradate by molecular hydrogen blisters. These are created by recombination of protons with metal electrons. Continued irradiation progresses blistering, which is undesired for many technical applications. In this work, the effect of the proton flux magnitude onto the degradation of native metal oxide layers and its consequences for blister formation has been examined. To study this phenomenon, we performed proton irradiation experiments of aluminium surfaces. The proton kinetic energy was chosen so that all recombined hydrogen is trapped within the metal structure. As a result, we discovered that intense proton irradiation increases the permeability of aluminium oxide layers for hydrogen atoms, thereby counteracting blister formation. These findings may improve the understanding of the hydrogen blistering process, are valid for all metals kept under terrestrial ambient conditions, and important for the design of proton irradiation tests.

npj Materials Degradation (2018)2:3; doi:10.1038/s41529-017-0024-z

\section{INTRODUCTION}

High vacuum and intense corpuscular radiation are among those environmental conditions that can change irreversibly the metal properties on a short timescale. Similar conditions are met in nuclear fusion reactor technologies. There, the so-called plasma facing materials are exposed to intense deuterium ion fluxes where the ion energy, of a few tens of eV, is released just below the metal surface. These metals, e.g., tungsten, suffer then from the formation of tiny blisters (few tens of $\mu \mathrm{m}$ in diameter) filled with molecular deuterium gas. ${ }^{1}$ Another field which opens new challenges to material science is space technology. There, materials have to withstand rapid temperature changes, intense corpuscular and electromagnetic radiation as well as impacts caused by space debris and micro-meteorites. Metals play a key role in space technology since they are used in many applications, e.g., for building primary structures of spacecrafts, as surface mirrors for space telescopes, as thin coatings deposited on thin elastic films (e.g., polyimide) used for insulation purposes to reflect light and propel solar sails. For space applications the blistering process causes e.g., a change of thermo-optical properties of metallic surfaces which can affect thermal properties of satellite interiors. In case of solar sailing, when the sail surface is populated by blisters, the effective momentum transfer from the solar photons is reduced by a drop of sail surface reflectivity. As a consequence, the propulsion abilities of the sail are reduced by the growing population of the blisters. ${ }^{2}$

In irradiation tests, the proton flux magnitude can be set freely from low to high values. The selected value is determined by the requirement to shorten laboratory time as much as possible to reach the number of incident protons which corresponds to the physical situation under study. The main motivation is to reduce the costs for maintenance of the facilities. However, such an approach has serious implications. An irradiation test should reveal the true nature of the changes in the material properties after the accumulation of the required proton number. This number is usually an equivalent of days, months or years during which the material is exposed to the respective environment. Certainly, the materials will change their physical properties in dependence on the applied proton energy and flux. The kinetic energy of the protons is released as heat in so-called thermal spikes. ${ }^{3}$ A larger proton flux and/or a larger proton kinetic energy will increase the number of thermal spikes. This, in turn, will augment the hydrogen diffusivity because the irradiation "drilled" holes into the test material structure. More holes enhance the mobility of hydrogen that can easier escape. This process can be named "radiation induced diffusion".

A standard of the European Cooperation for Space Standardisation stated that metals, e.g., aluminium, copper, nickel, titanium, and stainless steels "do not suffer from space-environment conditions" also that "radiation at the level existing in space does not modify the properties of metals". ${ }^{4}$ However, it has been shown that solar wind protons, while bombarding metallic surfaces, recombine with free electrons to hydrogen atoms within the metal structure. As a consequence surface blisters filled with molecular hydrogen gas are formed. ${ }^{5}$

In 2014, a new standard was published which states that metals can be embrittled by hydrogen. ${ }^{6}$ Hydrogen embrittlement corresponds to four corrosion mechanisms: formation of a hydride phase, enhanced local plasticity, grain boundary weakening, and blistering. ${ }^{7}$

Blistering is a fundamental process of metallic surface erosion. When taking place on metallic surfaces caused by exposure to a proton flux, ${ }^{8}$ the recombination processes ${ }^{9-11}$ of the protons with metal electrons to neutral hydrogen atoms are precondition for the blistering phenomenon. Hydrogen blisters occur as a consequence of supersaturation of hydrogen within the target material structure. ${ }^{12}$ The blisters change the physical properties of the exposed metals and increase their erosion rate. ${ }^{8}$

The growth of blisters depends strongly on environmental conditions, i.e., proton energy, integrated protons flux (hereafter called fluence), and sample temperature. ${ }^{13,14}$ However, the efficiency of the growth is also influenced by the number of

${ }^{1}$ DLR Institute of Space Systems, Mechanic and Thermal Systems, Robert-Hooke-Str. 7, 28359 Bremen, Germany; ${ }^{2}$ Institute of Physics, University of Zielona Góra, Szafrana 4a 65069 Zielona Góra, Poland and ${ }^{3}$ J. Gil Institute of Astronomy, University of Zielona Góra, Szafrana 2 65-516 Zielona Góra, Poland

Correspondence: Maciej Sznajder (Maciej.Sznajder@dlr.de)

Received: 21 August 2017 Revised: 16 November 2017 Accepted: 5 December 2017

Published online: 23 January 2018 
Table 1. Test parameters: sample symbol, irradiation time $t_{\text {irr }}$, proton fluence $F$, its uncertainty $\delta F$, as well as average blister radius $\langle R>$ and its uncertainty $\delta R$

\begin{tabular}{llllll}
\hline Sample & $\begin{array}{l}t_{\text {irr }} \times \\
10^{5}[\mathrm{~s}]\end{array}$ & $\begin{array}{l}F \times 10^{17} \\
{\left[\mathrm{p}^{+} \mathrm{cm}^{-2}\right]}\end{array}$ & $\begin{array}{l}\delta F \times \\
10^{17}\left[\mathrm{p}^{+} \mathrm{cm}^{-2}\right]\end{array}$ & $<R>[\mu \mathrm{m}]$ & $\delta R[\mu \mathrm{m}]$ \\
\hline S1 & 0.62 & 1.40 & \pm 0.003 & 0.112 & \pm 0.063 \\
S2 & 1.39 & 2.97 & \pm 0.007 & 0.136 & \pm 0.096 \\
S3 & 2.84 & 6.10 & \pm 0.015 & 0.154 & \pm 0.098 \\
S4 & 3.41 & 7.74 & \pm 0.019 & 0.126 & \pm 0.073 \\
S5 & 5.52 & 11.86 & \pm 0.029 & 0.132 & \pm 0.067 \\
S6 & 5.95 & 13.51 & \pm 0.033 & 0.090 & \pm 0.046 \\
S7 & 9.74 & 20.93 & \pm 0.051 & 0.095 & \pm 0.044 \\
S8 & 9.97 & 22.66 & \pm 0.055 & 0.099 & \pm 0.052 \\
S9*a & 9.41 & 12.81 & \pm 0.092 & - & - \\
S10*a & 3.92 & 12.77 & \pm 0.019 & - & - \\
\hline
\end{tabular}

a Sample S9* $^{*}$ was exposed to $\sim 1.5$ times smaller proton flux than the samples S1-8 while the sample $S 10^{*}$ was exposed to $\sim 1.6$ times larger proton flux than the samples S1-8.

impurities and defects within the target metal structure ${ }^{13}$ as well as its crystallographic orientation. ${ }^{15}$

The influence of the incident proton kinetic energy on blister growth mechanism in aluminium has been investigated experimentally. ${ }^{16}$ It has been found that kinetic energy of $70 \mathrm{keV}$ is a threshold below which the blisters populate aluminium surfaces at room temperature. ${ }^{16}$ For higher energies, an annealing procedure is necessary since the deposited hydrogen atoms are located too deep below the sample surface. This requires an additional heat input. Then, the hydrogen can diffuse closer to the surface and form the blisters.

Aluminium kept at room temperature starts to form surface blisters when the proton fluence exceeds $10^{16} \mathrm{p}^{+} \mathrm{cm}^{-2} .{ }^{16}$ The temperature of the irradiated metal has a strong influence on the blister growth mechanism. Two experimental approaches are possible. First, the test sample is kept at room or cryogenic temperature while being exposed to a proton flux. After reaching the required fluence, the sample will be annealed to tens or hundreds degrees Celsius. Second, the test material is bombarded by protons at already elevated temperatures. Both procedures lead to quantitatively different results. Irradiation of the test material, while realising the first experimental set-up, leads to a trapping of the recombined hydrogen atoms in vacancies within the metal structure. Then, during the annealing procedure, the hydrogen is released from the lattice sites and may form a high surface density of blisters. ${ }^{13,14,16,17}$ Exposing metals to the flux of protons at elevated temperatures leads to a continuous loss of hydrogen due to diffusion processes. Therefore, longer exposure times are required to reach the same hydrogen content within the metal structures compared to the first test scenario. Such a situation is realised when the plasma facing materials used in nuclear fusion reactors are exposed to deuterium ions. ${ }^{1}$ A similar situation appears if metallic surfaces react with solar wind ions in the interplanetary medium. ${ }^{2}$ For the here presented results, the examined metals were exposed to protons at elevated temperature.

The dependence of blister growth mechanism on the crystallographic orientation has been studied experimentally. It has been proven that for aluminium the blisters preferably form on $\{111\}$ instead on $\{100\}$ and $\{110\}$ planes. ${ }^{15}$

\section{RESULTS AND DISCUSSION}

There are three possibilities of implanting hydrogen into the test material, i.e., the gas plasma method, the electrochemical method, and the chemical method. Sample exposure to gas ions avoids the well-known problem of $\mathrm{Al}(\mathrm{OH})_{3}$-layer formation after performing the electrochemical or chemical charging of a test sample. ${ }^{18}$ Hence, we have used the gas plasma method to implant protons to the aluminium structure. Ten foil samples have been exposed to the proton flux. Irradiation time $t_{\text {irr }}$ accumulated fluence $F$, as well as its systematic errors are given in Table 1.

The test material is a metallised film, i.e., $7.5 \mu \mathrm{m}$ Upilex-S polyimide foil covered on both sides with $100 \mathrm{~nm}$ vacuum deposited aluminium (VDA) layers provided by the UBE Company. Since the foil was stored under room conditions (relative humidity $55 \pm 5 \%$, temperature $22 \pm 2{ }^{\circ} \mathrm{C}$ ) its surface is covered with a native AlOx layer, approx. $5 \mathrm{~nm}$ thick. ${ }^{19}$

Irradiation tests have been performed by use of a linear proton accelerator at the German Aerospace Center in Bremen. The accelerator is part of the so-called Complex Irradiation Facility (CIF) established to investigate the changes of physical material properties, caused by electromagnetic and corpuscular radiation.

The facility has been built in Ultra High Vacuum (UHV) technology and without rubber sealings to avoid selfcontamination. It is equipped with electron and proton linear accelerators, where both can generate independently particles in the energy range from 1 to $100 \mathrm{keV}$ with a maximum current of $100 \mu \mathrm{A}$. Three electromagnetic sources are also available, i.e., an argon VUV source, a deuterium and a xenon lamp, also called as a solar simulator. When working simultaneously they cover a wide wavelength range from $40 \mathrm{~nm}$ to $2150 \mathrm{~nm}$. The facility is also equipped with a mass-spectrometer which can analyse the particle spectrum coming out of a test object generated during an irradiation test. The facility is presented in Fig. 1. All beams of the radiation sources meet at the irradiation chamber where the test sample is placed.

The protons are generated by ionisation of the hydrogen gas stored in a bottle and placed within a secure deck close to the accelerator. The hydrogen is guided through a thermo-mechanical gas inlet valve with remote control to the ion source. The ionisation takes place within a glass bulb with a radio frequency, which is capacitively coupled to the bulb. The source output is optimised by control of the source gas pressure and oscillator loading. The plasma is then pushed out of the bulb to the acceleration section by a proper value of the so-called extraction voltage. Protons are accelerated in an appropriate tube by a high voltage, which corresponds to the required energy. The accelerator tube is manufactured as a metal to ceramic brazed assembly with no organic compounds. After the acceleration to beam line vacuum tubes which end within the irradiation chamber where a sample material under study is placed.

The irradiation procedure together with discussion of the error bars are given in the Supplementary Notes.

The outcome of the irradiation tests was unexpected. Based on previous experimental studies ${ }^{2}$ an increase of the average blister radius has been expected for larger proton fluences. However, it turned out that on samples which received the largest number of protons appear the smallest size and number of blisters.

In Fig. 2, the selected Atomic Force Microscope (AFM) images are shown which represent eight samples denoted as S1-8, respectively. Based on this figure, one may note that samples from $\mathrm{S} 1$ to S5 are populated with the highest number of blisters, while in the case of samples denoted as S6, S7, and S8 a considerable decrease of both the number and size of blisters can be observed.

Figure 3 shows magnified sample areas which in turn allow to see blisters in more detail. In particular, there are two samples which deserve special attention, i.e., samples denoted as S2 and S8. The sample S2 in Fig. $3 \mathrm{~b}$ has on its surface a region where the aluminium layer was mechanically removed before irradiation process and, therefore, no blisters have been formed on its surface. This area is marked by the white rectangle. Sample S8 (in Fig. 3h) indicates two special features. The first of them 


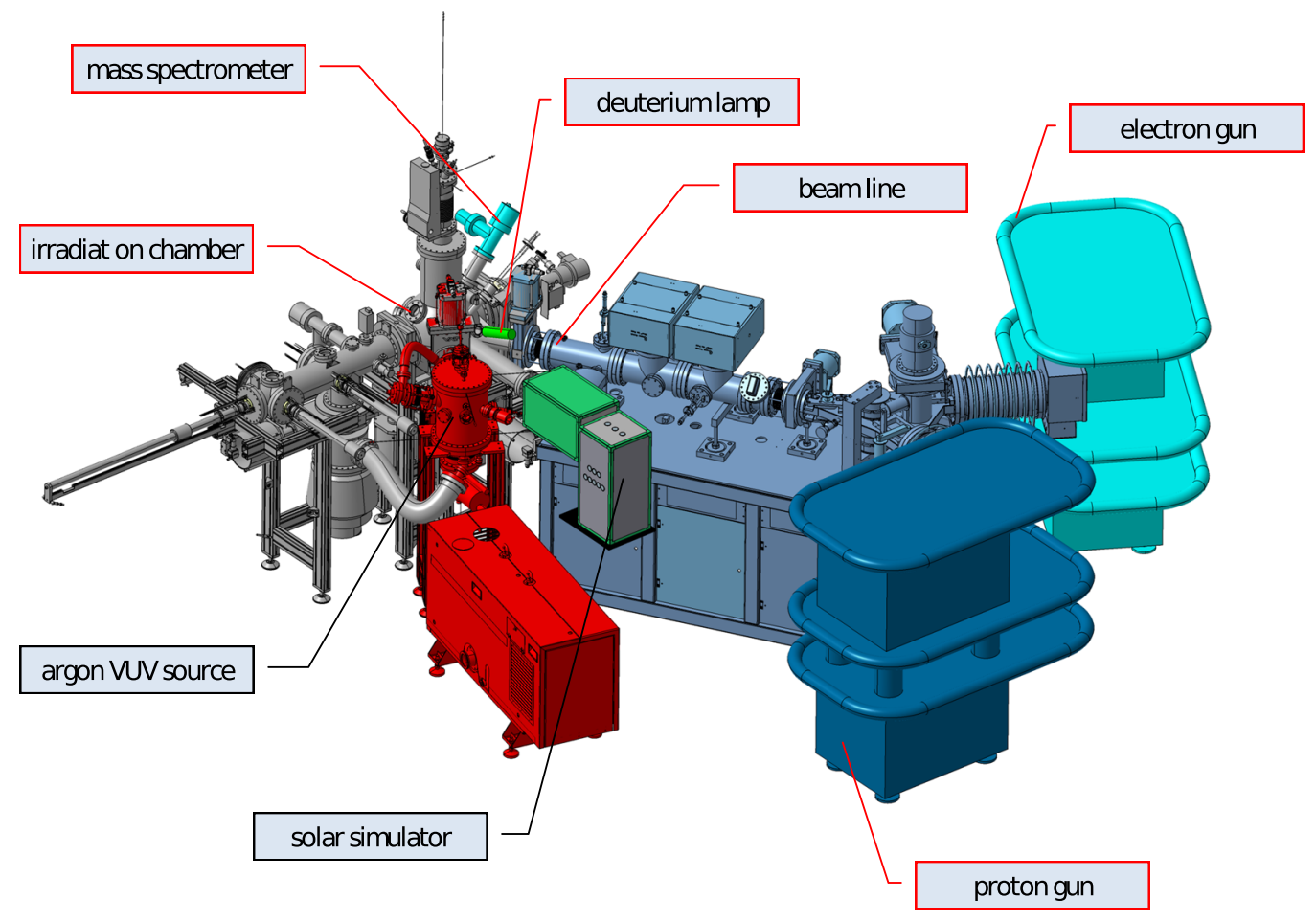

Fig. 1 Schematic representation of radiation sources of the complex irradiation facility

corresponds to a long exposure of the sample to the proton flux which results with AlOx surface delamination (white circle) and the second effect which is associated with the crack of a bubble cup (shown within a small white-bordered window). The influence of the proton fluence on the blister growth is discussed in the following paragraphs.

The blister population in S1-8, was studied by help of digital processing of the corresponding AFM images shown in the Fig. 2 (see Methods). In this approach, the images were converted into a grid composed of black and white pixels and the Hoshen-Kopelman clustering algorithm ${ }^{20}$ was used to discriminate blisters and to calculate their average radius $\langle R>$.

The outcome of the studies of $\mathrm{S} 1-8$, suggests that the evolution of blisters can be classified into three stages (marked with different colours, see Fig. 4b). They are all determined by the degradation level of the native AlOx-layer.

Figure $4 a$ shows the relation between blister numbers and their radii. Special attention was given to find out how the average blister radius evolves with increasing irradiation time and how it affects the number of blisters. This is visualised in Fig. $4 \mathrm{~b}$. The radii and their error bars are given in the last two columns of Table 1. The parameter $\delta \mathrm{R}$ denotes standard deviation which is a measure of how spread out the values of blister radii are. Figure $4 c$ displays the relation between blister number and irradiation time. In general, the longer the irradiation time, the smaller the number of blisters. An exception is sample S7 where the number of blisters exceeds those present on e.g., sample S8.

The dependence of the average blister radius $<R>$ on exposition time of the aluminium foil to proton flux is presented in Fig. $4 \mathrm{~b}$. It suggests that blistering is a discontinuous process. Initially, excess hydrogen agglomerate at the interface between aluminium and the protective oxide layer; ${ }^{15,19,21}$ stage marked with green colour. The hydrogen is also collected in different kinds of defects which are natural traps for diffusing interstitial atomic hydrogen. ${ }^{22-24}$ The increase of internal hydrogen gas pressure is the reason for the observed blister growth. The appearance of a large excess of hydrogen at the AlOx/Al interface may lead to Al surface reconstruction through the faceting growth mechanism. This growth stage of hydrogen blisters (marked with yellow colour) is interrupted by detachment of the AlOx layer from the internal aluminium layer. In this situation, large pieces of the oxide layer can be removed. The third stage of the blistering process seen in Fig. 4c (marked with red colour) takes place when the oxide layer is mostly degraded. In the former work of the authors, ${ }^{2}$ the applied proton flux was weaker than here. Then, the evolution of hydrogen blisters is a continuous process and their average radius $<R>$ increases in accordance to a particular power law.

The progressing degradation of the AlOx layer can explain the decreasing blister number while the samples receive larger proton fluences (Fig. 4c). The hydrogen permeability increases while the sample surface is scarred with a larger number of cracks. Therefore, a smaller amount of hydrogen within the aluminium structure can fuel the blisters and, consequently, a smaller number of blisters appear on the surface.

The dependency of the AlOx ageing on the proton flux has been examined too. Three samples, i.e., S9*, S6, and $\mathrm{S} 10^{*}$ have been exposed to three different proton fluxes: $1.36 \times 10^{12}, 2.27 \times 10^{12}$, and $3.26 \times 10^{12} \mathrm{p}^{+} \mathrm{cm}^{-2} \mathrm{~s}^{-1}$, respectively. Here, a Field Emission Microscope (FEM) has been used to analyse the samples. All three samples received comparable proton fluences, see Table 1.

Sample S9* has been exposed to the lowest proton flux, see Figs. $5 a$, b. Here, small dark areas can be spotted. These are places where the AlOx layer has been delaminated from the aluminium substrate (marked with blue circles).

Sample S6 received an intermediate proton flux. Here, the presence of small delamination centres but also large surface areas delaminated from the aluminium substrate are seen (marked with green circles, Figs. 5, d). One can also recognise structures which appear as crack-lines formed at the AlOx surface (marked with red dashed lines). They are thin dark intersecting lines, covering the sample area exposed to protons.

Sample $\mathrm{S} 10^{*}$ received the highest proton flux (Figs. 5e, f). Clearly, there the AlOx layer is cracked. Figure $5 \mathrm{e}$ shows in particular a blister cup scared with the crack-lines, while at Fig. $5 f$ a 

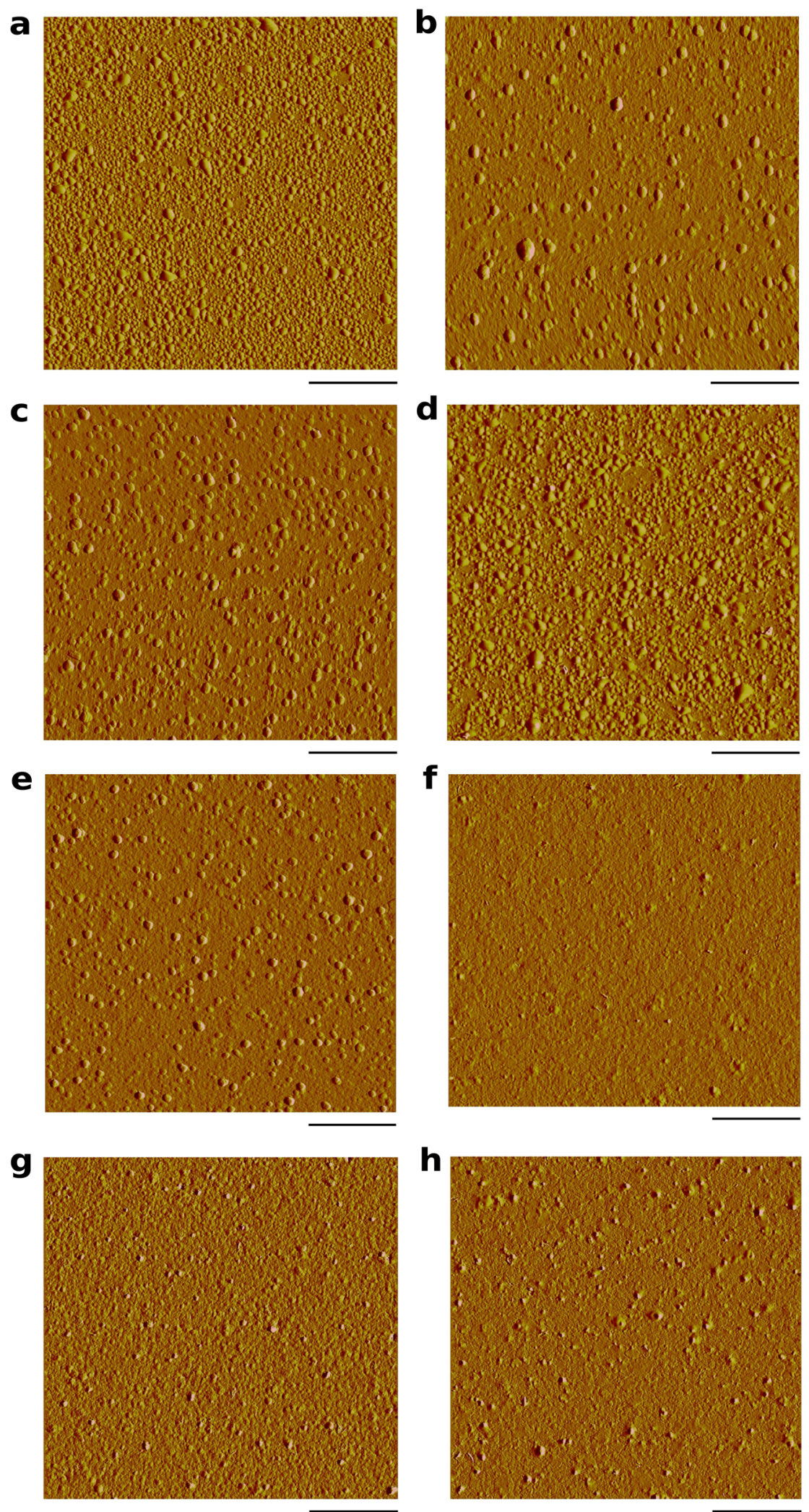

Fig. 2 Surface morphology of the specimens for eight different proton fluences. a, Sample S1. b, Sample S2. c, Sample S3. d, Sample S4. e, Sample S5. f, Sample S6. g, Sample S7. h, Sample S8. All scale bars, $5 \mu \mathrm{m}$. Photographs were taken with AFM

large crack located at the base of a blister is seen. Note that the delamination of the AlOx layer (marked with white circle, Fig. 3h), as well as cracking of the blisters (seen in white rectangular window, Fig. 3h) have been observed for the sample S8 as well. It proofs the progressing material degradation and its influence on the blistering process.

The considered proton fluxes and energies can be compared with proton fluxes present in space. The chosen kinetic energy of 


\section{a}

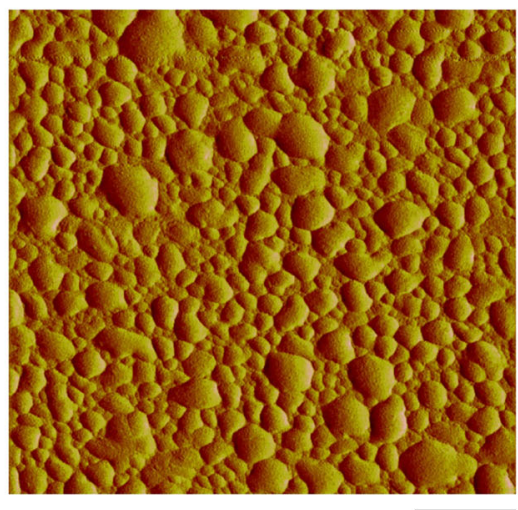

C

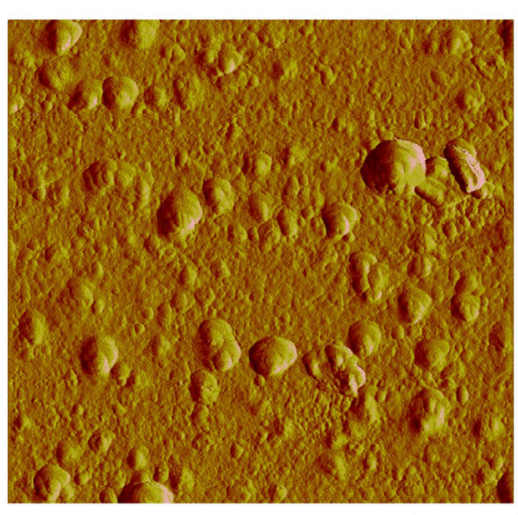

$\mathbf{e}$

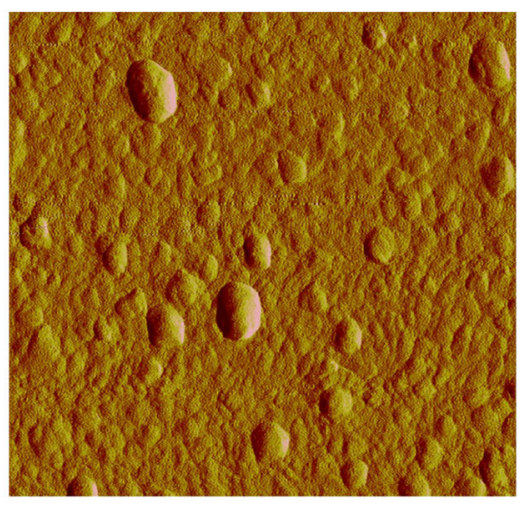

$\mathbf{g}$

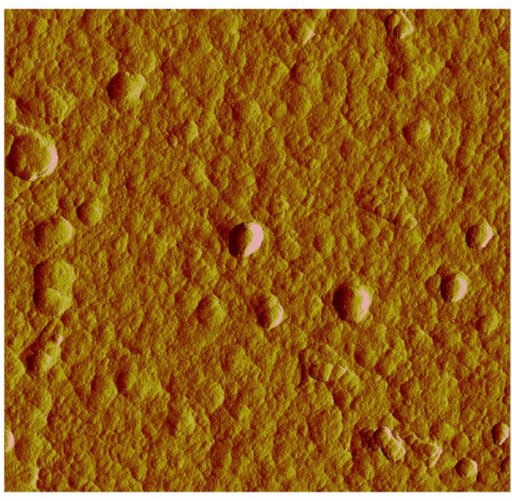

b

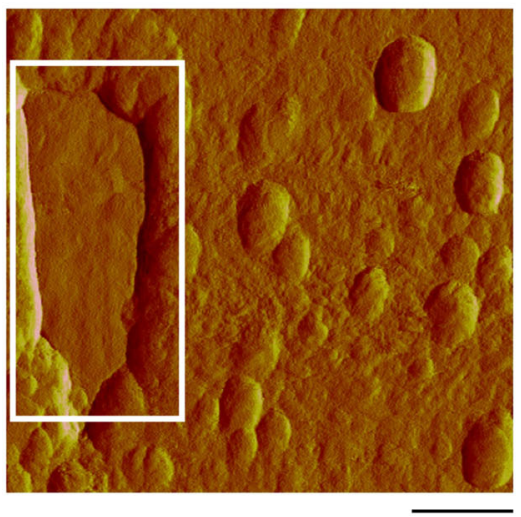

d

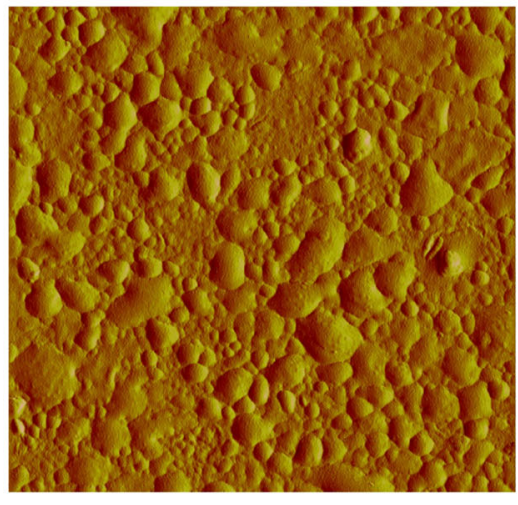

$f$

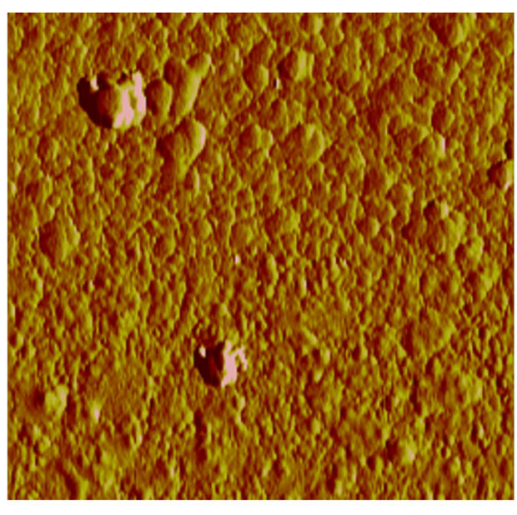

h

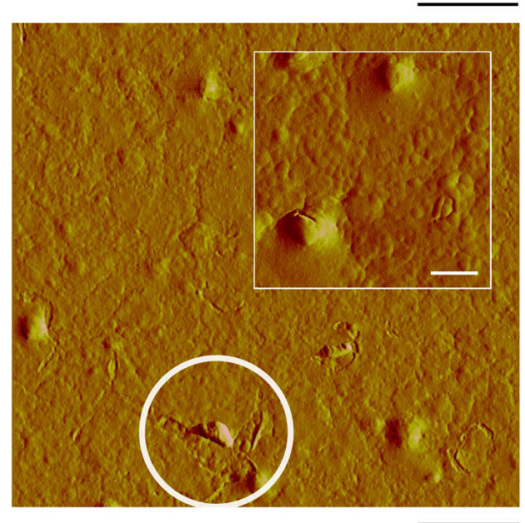

Fig. 3 Surface morphology of the specimens for eight different proton fluences. a, Sample S1. b, Sample S2. c, Sample S3. d, Sample S4. e, Sample S5. f, Sample S6. g, Sample S7. h, Sample S8. Black scale bars, $1 \mu \mathrm{m}$. White scale bar, $0.3 \mu \mathrm{m}$. Photographs were taken with AFM

the protons $(2.5 \mathrm{keV})$ was intended to deposit the recombined hydrogen completely within the aluminium. In the close vicinity of the Earth, i.e., up to altitudes of the geostationary orbit, the recorded proton fluxes start from energies of approx. $50 \mathrm{keV}$.
However, for the interplanetary space the available data start from energy of few eVs.

In the interplanetary space a test object is subjected to a wide energy spectrum of the protons, from eVs up to hundreds of 
MeVs. The $100 \mathrm{~nm}$ aluminium would collect protons up to energy of $8 \mathrm{keV} .{ }^{25}$ Protons with higher energies travel through the aluminium and may stop within the polyimide substrate of the foil. They are of no interest, since they do not recombine to hydrogen, and therefore, they provide no contribution to the blister formation. A typical proton flux in the solar wind at 1 astronomical unit (AU) distance from the Sun and energy lower than $10 \mathrm{keV}$ is $\sim 3 \times 10^{8} \mathrm{p}^{+} \mathrm{cm}^{-2} \mathrm{~s}^{-1}$. ${ }^{26}$ The flux can be estimated using e.g., the Advanced Composition Explorer (ACE) database. ${ }^{27,28}$ Taking the data from the lowest recorded energy to an energy of $8 \mathrm{keV}$ in steps of $1 \mathrm{eV}$ the cumulative flux is $\sim 2 \times 10^{12}$ $\mathrm{p}^{+} \mathrm{cm}^{-2} \mathrm{~s}^{-1}$. It is comparable to the flux to which the samples S1-8 has been exposed. Sample $S 9^{*}$ received an about 1.5 times smaller flux while that of sample $\mathrm{S} 10^{*}$ is nearly 1.6 times larger.

The experiments show clearly that the proton flux magnitude dictates the blister evolution when a native AIOx layer is formed on aluminium. It cracks and delaminates when the sample surface is bombarded by a flux of $2.27 \times 10^{12} \mathrm{p}^{+} \mathrm{cm}^{-2} \mathrm{~s}^{-1}$ or higher. For lower fluxes the native AlOx is delaminated from its aluminium substrate as well, however on relatively small areas (Figs. 5a, b). The evolution of blisters can be classified into three stages. First, the blisters grow most dynamically and have dimensions of up to $0.3 \mu \mathrm{m}$ in diameter before the AlOx starts to delaminate or crack. Second, the AlOx degrades and loses its ability to hold hydrogen within the aluminium structure. This results in blister diameters of about $0.25 \mu \mathrm{m}$. Third, if the aluminium is bombarded furthermore incessantly by protons, the process of AlOx degradation continues. Then, the typical blister diameter is about $0.2 \mu \mathrm{m}$.

In the context of space applications, proton fluxes received by the here shown samples represent their state at $1 \mathrm{AU}$ from the Sun. After only a few days under space conditions the aluminium foil would be covered by hydrogen blisters as shown in the samples S1-8. A small increase of the flux by a factor of $\sim 1.6$ causes a significant change in the aluminium surface morphology (sample S10*). Hence, the radiation driven blistering phenomenon is very sensitive to process parameters.

These results are important for the planning of proton irradiation tests, especially for the well-suited selection of the flux magnitude. A careful choice of the applied proton flux is necessary. Too high fluxes will lead to an acceleration of material degradation that does not reflect the true nature of ageing processes as take place in the environment under study.

\section{METHODS}

In this work, two microscopy methods were used to visualise the blisters, an Atomic Force Microscope (AFM-Bioscope Catalyst by Bruker) and a Field Emission Microscope (FEM-FE-REM, HITACHI 4004 with EDX-System EDAX Genesis 5).

In order to study in more detail the blister population, the blisters were counted and their radius was measured. This has been achieved by digital processing of the AFM images. They show different blistering stages at the aluminium foil surface. In order to approximate the average blister size the image data were converted first into ppm format (portable pixmap format) providing a map of $\mathrm{N} \times \mathrm{M}$ colour pixels (defined by a RGB triplet of integers, each of them ranging from 0 to 255). They were next converted into black and white pixels-the pixel colour was replaced by black if the RGB sum was less than a threshold value of 459 and the white colour otherwise. In this study, the Hoshen-Kopelman algorithm ${ }^{20}$ for labelling clusters on a grid composed of black and white pixels was used to estimate the average radius $\langle R>$ of blisters. Clusters representing blisters were defined by the collection of these black pixels which were in the nearest-neighbour relationship on the bitmap grid under consideration.

Data availability

The data presented and discussed in this study is available from the authors upon reasonable request. a

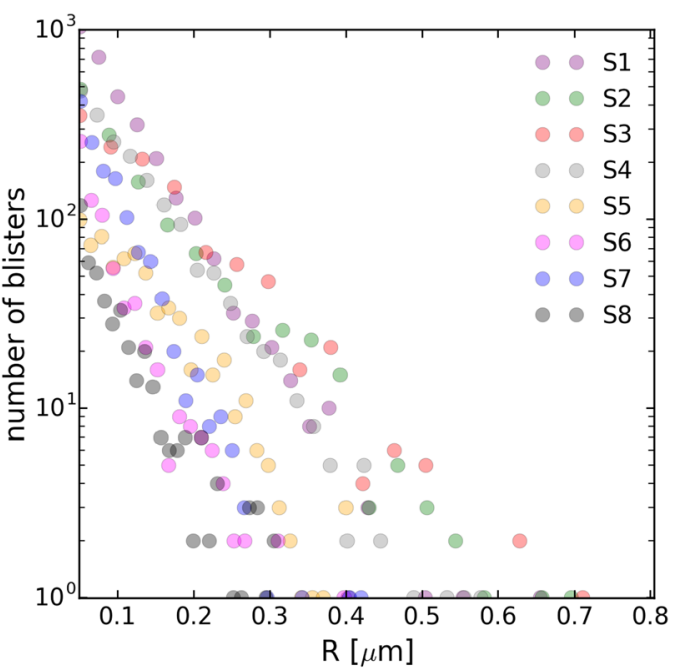

b

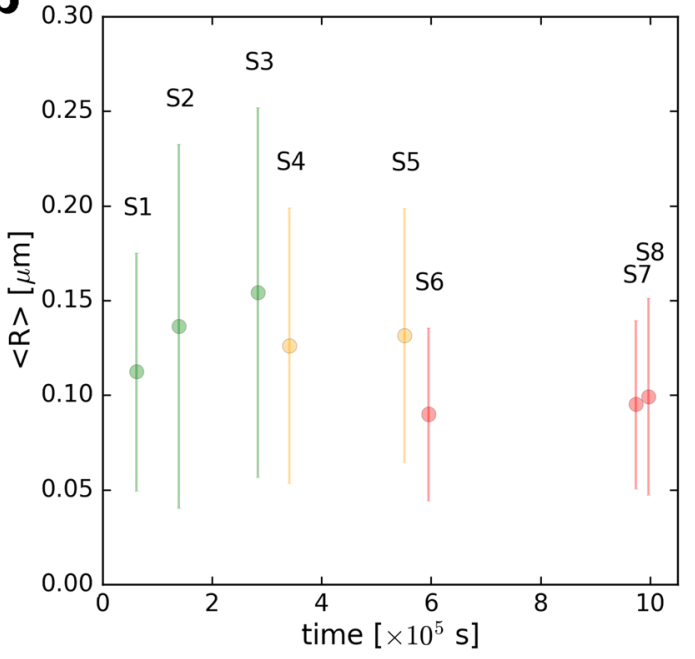

C

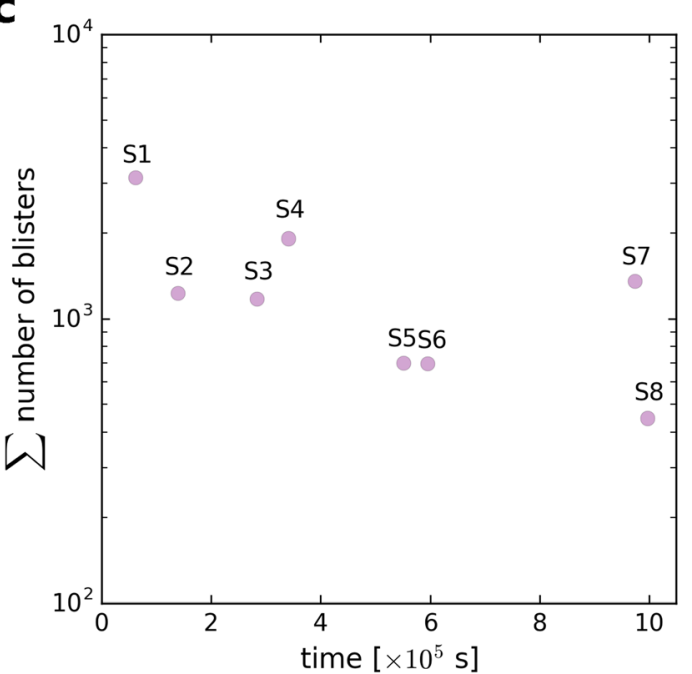

Fig. 4 a, Number of blisters as function of their radii. $\mathbf{b}$, average blister radius of the population as function of the irradiation time. $\mathbf{c}$, recap number of blisters assigned to the irradiation time. Analysis was based on samples S1-8 pictures taken from Fig. 2 

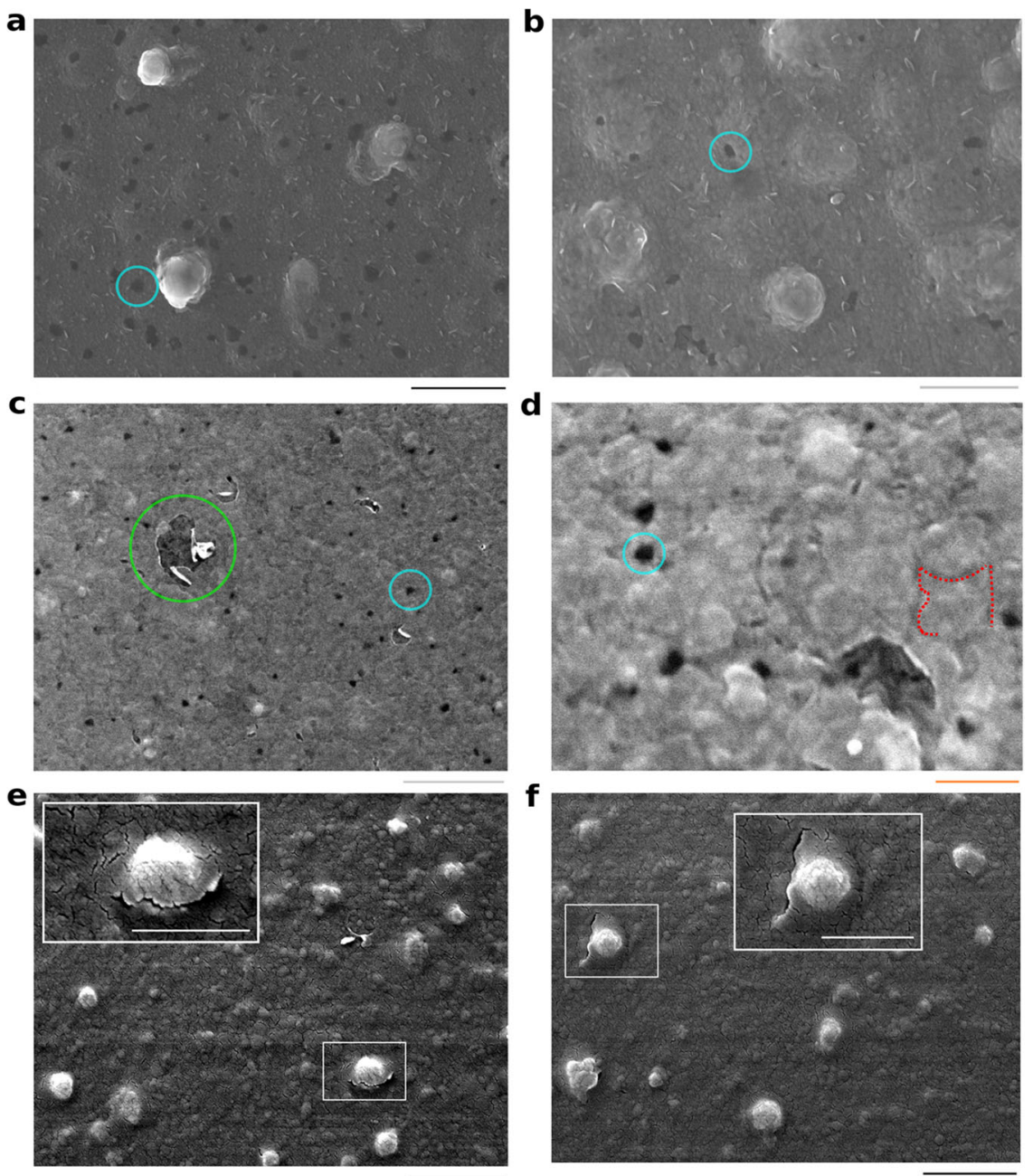

Fig. 5 Morphology of samples exposed to three different proton fluxes: $1.36 \times 10^{12}$ (top row), $2.27 \times 10^{12}$ (middle row), and $3.26 \times 10^{12}$ $\mathrm{p}^{+} \mathrm{cm}^{-2} \mathrm{~s}^{-1}$ (bottom row). Specimens receive similar proton fluence. First row, sample S9* $\mathbf{a}$ and $\mathbf{b}$, small number of AlOx delamination areas are present on the sample surface exposed to protons. Second row, sample S6 c and d, crack lines as well as delamination centres cover the irradiated area. Third row, sample $\mathrm{S} 10^{*} \mathbf{e}$ and $\mathbf{f}$, crack lines dominate the exposed areas. Black line: scale bars, $1 \mu \mathrm{m}$. Grey line: scale bars, 0.7 $\mu \mathrm{m}$. White line: scale bars, $0.5 \mu \mathrm{m}$. Orange line: scale bar, $0.2 \mu \mathrm{m}$. Photographs were taken with FEM

\section{ACKNOWLEDGEMENTS}

The authors acknowledge Mr. Thomas Renger who commissioned the Complex Irradiation Facility that used to obtain the experimental data. The authors like to thank the IWT Stiftung Institut für Werkstofftechnik located in Bremen, Germany for performing the FEM microscope analysis and the Science and Technology Park University of Zielona Góra, Poland for performing the AFM microscope analysis.

\section{AUTHOR CONTRIBUTIONS}

M.S. conducted and performed the proton irradiation experiments. M.D. processed the AFM images with the Hoshen-Kopelman algorithm. All authors contributed equally both with discussion of the results and with writing the paper.

\section{ADDITIONAL INFORMATION}

Supplementary information accompanies the paper on the npj Materials Degradation website (https://doi.org/10.1038/s41529-017-0024-z).

Competing interests: The authors declare no competing financial interests.

Publisher's note: Springer Nature remains neutral with regard to jurisdictional claims in published maps and institutional affiliations.

\section{REFERENCES}

1. Shu, W. M., Wakai, E. \& Yamanishi, T. Blister bursting and deuterium bursting release from tungsten exposed to high fluences of high flux and low energy deuterium plasma. Nucl. Fusion 47, 201-209 (2007).

2. Sznajder, M., Geppert, U. \& Dudek, M. Degradation of metallic surfaces under space conditions, with particular emphasis on Hydrogen recombination processes. Adv. Space Res. 56, 71-84 (2015).

3. Szenes, G. Comparison of two thermal spike models for ion-solid interactions. Nucl. Instrum. Methods Phys. Res. B 269, 174-179 (2011).

4. European Cooperation for Space Standardization ECSS-Q-70-71A rev. 1 Space product assurance: Data for selection of space materials and processes. (ESA-ESTEC Requirements and Standards Division, 2004).

5. Sznajder, M. \& Geppert, U. H2 blister formation on metallic surfaces-a candidate 308 for degradation processes in space. Adv. Solar Sailing. Springer, pp 559-571 (ISBN: 978-3-642-34907-2) (2014).

6. European Cooperation for Space Standardization ECSS-E-ST-32-08C Rev.1; Space engineering: Materials (ESA Requirements \& Standards Division, 2014).

7. Myers, S. M. et al. Hydrogen interactions with defects in crystalline solids. Rev. Mod. Phys. 64, 559-617 (1992).

8. Astrelin, V. T. et al. Blistering of the selected materials irradiated by intense 200 keV proton beam. J. Nucl. Mater. 396, 43-48 (2010).

9. Eichler, J. Lectures on ion-atom collisions, from nonrelativistic to relativistic velocities. Chapter 10 (Elsevier B. V., 2005).

10. Hagstrum, H. D. Theory of Auger ejection of electrons from metals by ions. Phys. Rev. 96, 336-365 (1954). 
Hydrogen blistering under extreme radiation M Sznajder et al.

11. Sols, S. \& Flores, F. Charge transfer processes for light ions moving in metals. Phys. Rev. B 30, 4478-4880 (1984).

12. Condon, J. B. \& Schober, T. Hydrogen bubbles in metals. J. Nucl. Mater. 207, 1-24 (1993).

13. Daniels, R. D. Correlation of hydrogen evolution with surface blistering in protonirradiated aluminium. J. Appl. Phys. 42, 417-419 (1971).

14. Milcius, D., Pranevicius, L. L. \& Templier, C. Hydrogen storage in the bubbles formed by high-flux ion implantation in thin Al films. J. Alloy. Compd. 398, 203-207 (2005).

15. Xie, D. G., Wang, Z. J., Sun, J., Li, J., Ma, E. \& Shan, Z. W. In situ study of the initiation of hydrogen bubbles at the aluminium metal/oxide interface. Nat. Mater. 14, 899-903 (2015).

16. Milacek, L. H., Daniels, R. D. \& Cooley, J. A. Proton-radiation-induced blistering of aluminum. J. Appl. Phys. 39, 2803-2815 (1968).

17. Kuznetsov, A. S., Gleeson, M. A. \& Bijkerk, A. Temperature dependencies of hydrogen-induced blistering of thin film multilayers. J. Appl. Phys. 115, 173510-173520 (2014).

18. Buckley, C. E. \& Birnbaum, H. K. Characterization of the charging techniques used to introduce hydrogen in aluminum. J. Alloy. Compd. 330-332, 649-653 (2002)

19. Evertsson, J. et al. The thickness of native oxides on aluminum alloys and single crystals. Appl. Surf. Sci. 349, 826-832 (2015).

20. Koshen, J. \& Kopelman, R. Percolation and cluster distribution. I. Cluster multiple labeling technique and critical concentration algorithm. Phys. Rev. B 14, 3438-3445 (1976).

21. Li, M., Xie, D. G., Ma, E., Li, J., Zhang, X.-X. \& Shan, Z.-W. Effects of hydrogen on the integrity of aluminium-oxide interface at elevated temperatures. Nat. Commun. 8, 14564-14571 (2017).

22. Greenwood, G. W., Foreman, J. E. \& Rimmer, D. E. The role of vacancies and dislocations in the nucleation and growth of gas bubbles in irradiated fissile materials. J. Nucl. Mater. 4, 305-324 (1959).

23. Lu, G. \& Kaxiras, E. Hydrogen embrittlement of aluminum: the crucial role of vacancies. Phys. Rev. Lett. 94, 155501-155504 (2005).
24. Xie, D. G., Li, S., Li, M., Wang, Z., Gumbsch, P., Sun, J., Ma, E., Li, J. \& Shan, Z. Hydrogenated vacancies lock dislocations in aluminium. Nat. Commun. 7, 13341 (2016).

25. Berger, M. J., Coursey, J. S., Zucker, M. A. \& Chang, J. ESTAR, PSTAR, and ASTAR: computer programs for calculating stopping-power and range tables for electrons, protons, and helium ions (version 2.0.1). http://physics.nist.gov/Star. National Institute of Standards and Technology. (2017).

26. Holzer, T. E. Interaction between the solar wind and the interstellar medium. Annu. Rev. Astron. Astrophys. 27, 199-234 (1989).

27. Chiu, M. C., Von-Mehlem, U. I., Willey, C. E., Betenbaugh, T. M. \& Maynard, J. J. et al. ACE Spacecraft. Space Sci. Rev. 86, 257-284 (1998).

28. ACE Science Center. California Institute of Technology, http://www.srl.caltech. edu/ACE/. (2017).

(C) Open Access This article is licensed under a Creative Commons Attribution 4.0 International License, which permits use, sharing, adaptation, distribution and reproduction in any medium or format, as long as you give appropriate credit to the original author(s) and the source, provide a link to the Creative Commons license, and indicate if changes were made. The images or other third party material in this article are included in the article's Creative Commons license, unless indicated otherwise in a credit line to the material. If material is not included in the article's Creative Commons license and your intended use is not permitted by statutory regulation or exceeds the permitted use, you will need to obtain permission directly from the copyright holder. To view a copy of this license, visit http://creativecommons. org/licenses/by/4.0/.

(c) The Author(s) 2018 